EdUCACIÓN VETERINARIA

\title{
Estilos de Aprendizaje de Estudiantes y Docentes de Primery Segundo Año de la Carrera de Medicina Veterinaria en Concepción, Chile
}

\author{
Learning Styles of Students and Teachers in First and Second Year of Veterinary \\ Medicine in Concepción, Chile
}

\begin{abstract}
Alvaro Luzio Q. ${ }^{1.4}$, Francisco Araneda S.M. ${ }^{1}$, Jacqueline Salgado A. ${ }^{2}$, Manuel Rain $\mathbf{F}^{3}$
\end{abstract}

\section{Resumen}

\begin{abstract}
Los enfoques pedagógicos actuales le entregan al estudiante un rol activo en los procesos de aprendizaje. El docente es un facilitador que interviene y colabora en los procesos de aprendizaje. En este sentido, los estilos de aprendizaje surgen como una herramienta pedagógica valiosa tanto para el alumno como para el docente. Estos se encuentran estrechamente relacionados con la forma en que los estudiantes aprenden, los profesores enseñan y cómo ambos interactúan en la relación de enseñanza-aprendizaje. Desde el punto de vista de una propuesta pedagógica, el modelo de estilos de aprendizaje de Kolb los clasifica en Activo, Reflexivo, Teórico y Pragmático. El presente trabajo plantea el estudio de los estilos de aprendizaje de estudiantes y docentes de primer y segundo año de la carrera de Medicina Veterinaria, Universidad Santo Tomás, en Concepción, Chile, mediante la aplicación del 'Cuestionario Honey y Alonso de estilos de aprendizaje' (CHAEA). Se aplicó el cuestionario a toda la población de estudiantes $(n=39)$ y docentes $(n=17)$ de los dos primeros años de la carrera. Los resultados indicaron una moderada preferencia hacia el estilo de aprendizaje reflexivo, tanto en estudiantes como en docentes, pero sin diferencias estadísticas entre estilos de aprendizaje.
\end{abstract}

Palabras clave: estilos de aprendizaje, estudiantes, docentes, medicina veterinaria

\footnotetext{
${ }^{1}$ Escuela de Medicina Veterinaria, Facultad de Recursos Naturales y Medicina Veterinaria, Universidad Santo Tomás, Concepción, Chile

${ }^{2}$ Centro de Aprendizaje, Universidad Santo Tomás, Concepción, Chile

${ }^{3}$ Escuela de Educación Básica, Facultad de Educación, Universidad Santo Tomás, Concepción, Chile

${ }^{4}$ E-mail:aluzio@santotomas.cl
} 
The current pedagogical approaches place in the student an active role in the learning process. The teacher is a facilitator who intervenes and helps in the learning process. In this sense, the learning styles emerge as a valuable pedagogical tool for both students and for teachers. They are closely related to the way students learn, to the teachers teaching style and how both interact in the teaching-learning relationship. From the point of view of a pedagogical approach, the model of Kolb's learning styles classifies them as Active, Reflective, Theoretical, and Pragmatic. The present study identifies the learning styles of students and teachers in the first and second year of Veterinary Medicine at the University of Santo Tomas, in Concepción, Chile by applying the Honey and Alonso learning styles questionnaire (CHAEA). The questionnaire was applied to all students $(n=39)$ and teachers $(n=17)$ of the first two years of the career. The results indicated a moderate preference towards reflective learning style on students and teachers; however, no statistical difference was found between learning styles.

Key words: learning styles, students, teachers, veterinary medicine

\section{INTRODUCCIÓN}

La enseñanza debe estar al servicio de la educación, involucrando aspectos que van más allá de la transmisión de información y conocimientos, potenciando en el proceso formativo el desarrollo de habilidades y actitudes que capaciten al estudiante para tener un rol activo en su aprendizaje. Para que un estudiante aprenda es necesario tomar en cuenta sus conocimientos e ideas previas, sus necesidades, expectativas, estilos y estrategias de aprendizaje (Ahumada, 2005).

Epistemológicamente han existido dos grandes posiciones sobre el origen del aprendizaje y su relación con el medio: por un lado el racionalismo que sustenta en sus grandes representantes, Platón, Descartes y Kant, que hay un mundo externo del que obtenemos información sensorial; sin embargo, las ideas se originan por obra de la mente, es decir, la razón actúa sobre los datos obtenidos del mundo (Tarpy, 1999). Por el otro lado, el empirismo sostiene, en sus representantes Aristóteles y Locke, que la experiencia es la única forma de conocimiento, por lo que al nacer nuestra mente se encuentra en blanco y las ideas se adquieren de la experiencia sensorial y de la reflexión de estas (Tarpy, 1999).

Es un hecho que los estudiantes son distintos, que poseen variados tipos de personalidad y que no todos aprenden de la misma manera ni al mismo ritmo por cuanto cada uno utiliza su propio método y estilo de aprendizaje (Acevedo y Rocha, 2011). Investigaciones recientes van dejando constancia de que los estilos de aprendizaje están estrechamente relacionados con la forma en que los estudiantes aprenden, los profesores enseñan y como ambos interactúan en la relación de enseñanza-aprendizaje (Adán, 2002). Es por esto que el concepto de estilos de aprendizaje es utilizado en las investigaciones psicopedagógicas actuales, debido a su valor teórico y metodológico para comprender y perfeccionar el proceso de enseñanza-aprendizaje en los diferentes niveles educativos por los que transitan los alumnos (Juárez et al., 2012, Pantoja et al., 2013).

Keefe (1988) (citado por Alonso et al., 1997) define los estilos de aprendizaje como «...los rasgos cognitivos, afectivos y fisiológicos que sirven como indicadores relativamente estables, de cómo los alumnos perciben, interaccionan y responden a sus ambien- 
Cuadro 1. Descripción de los estilos de aprendizaje según modelo de Honey y Mumford (1986)

\begin{tabular}{llll}
\hline Activo & Reflexivo & Teórico & Pragmático \\
\hline $\begin{array}{l}\text { Experiencias nuevas } \\
\text { Disfrutan el presente }\end{array}$ & $\begin{array}{l}\text { Observadores } \\
\text { Analíticos }\end{array}$ & $\begin{array}{l}\text { Lógicos y racionales } \\
\text { Integran las } \\
\text { observaciones en } \\
\text { teorías complejas y } \\
\text { coherentes } \\
\text { Piensan en forma } \\
\text { secuencial }\end{array}$ & $\begin{array}{l}\text { Pragmáticos } \\
\text { Realistas }\end{array}$ \\
$\begin{array}{l}\text { Entusiastas ante lo } \\
\text { nuevo }\end{array}$ & Precavidos & $\begin{array}{l}\text { Decisiones rápidas } \\
\text { de la resolución de } \\
\text { problemas }\end{array}$ \\
$\begin{array}{l}\text { Rodeados de gente, } \\
\text { pero ellos son el } \\
\text { centro de las } \\
\text { actividades }\end{array}$ & $\begin{array}{l}\text { Arriban a } \\
\text { conclusiones } \\
\text { después de análisis } \\
\text { detallado }\end{array}$ & $\begin{array}{l}\text { Ajenos los juicios } \\
\text { subjetivos }\end{array}$ & $\begin{array}{l}\text { Se impacientan } \\
\text { con discusiones } \\
\text { extensas sobre una } \\
\text { misma cuestión }\end{array}$ \\
\hline
\end{tabular}

tes de aprendizaje...». Por otra parte, Dunn R y Dunn K (1984) los definen como la forma en la que los estudiantes se concentran, procesan, internalizan y recuerdan información académica nueva. El estilo de aprendizaje indica una preferencia de la persona a enfocar sus mecanismos cognitivos hacia determinados tipos de selección, percepción y comprensión de la información, el cual se caracteriza por variar de acuerdo a las situaciones (Pantoja et al., 2013). Conocer el estilo personal de aprendizaje aporta al estudiante una guía para comprender el modo en que tiende a usar algunas de sus características personales en las distintas fases del proceso cíclico de aprender; además, le facilita la toma de conciencia de aquellas destrezas y conocimientos que ha de poseer para poder aprender de manera efectiva en cualquier situación y alcanzar el éxito en lo que está aprendiendo (Alonso et al., 1997).

El aprendizaje es un proceso cíclico entendido como construcción de la experiencia $y$, para que sea eficaz, necesita de cuatro etapas: experiencia concreta, observación reflexiva, conceptualización abstracta y experimentación activa (Witkin y Goodenoug,
1991). Esto supone experimentar, reflexionar, elaborar hipótesis y verificarlas. Idealmente, un aprendiz eficaz presenta capacidades para cada una de ellas; sin embargo, lo común es que seamos capaces de alguna de ellas. Esta mayor capacidad se refleja en nuestra preferencia por aprender de un modo consistente con nuestra preferencia. El estilo no guarda relación directa con las aptitudes cognitivas generales, sino más bien con su historia personal (Alonso et al., 1997). Existen modalidades y peculiaridades personales para aprender, pero ¿se pueden diagnosticar?, ¿se pueden cambiar los estilos?, ¿de qué dependen?, ¿cómo se clasifican?, ¿qué implicaciones pedagógicas se deducen? (Alonso et al., 1997).

Kolb, en 1989, desarrolló un instrumento para reconocer y medir las fortalezas y debilidades de estas capacidades en los estudiantes. Basado en estos trabajos, Honey y Mumford (1986), con base a la teoría de Kolb, propusieron un modelo de estilos de aprendizaje con una descripción detallada de los estilos basada en la acción de los estudiantes: activo, reflexivo, teórico y pragmático (Cuadro 1). Asimismo, estos autores crearon el «Learning Styles Questionnaire» (LSQ), que 
Cuadro 2. Promedios \pm desviaciones estándar de los puntajes por estilo de aprendizaje del 'Cuestionario Honey Alonso de Estilos de Aprendizaje' (CHAEA) aplicado a los estudiantes $(n=39)$ y docentes $(n=17)$ de primer y segundo año de la carrera de Medicina Veterinaria en Concepción, Chile

\begin{tabular}{ccccc}
\hline & Activo & Reflexivo & Teórico & Pragmático \\
\hline Estudiantes & $12.8 \pm 2.95$ & $14.8 \pm 3.09$ & $13.2 \pm 2.92$ & $13.3 \pm 2.79$ \\
Docentes & $9.4 \pm 2.7$ & $16.1 \pm 1.6$ & $15 \pm 2.5$ & $12.1 \pm 2.2$ \\
\hline
\end{tabular}

fue posteriormente adaptado al idioma español por Alonso et al. (1997). El instrumento 'Cuestionario Honey y Alonso de estilos de aprendizaje' (CHAEA), de Alonso y Gallego, es el más utilizado en el idioma español y se ha empleado en diversas investigaciones desde 1992 (García et al., 2009), en países tales como Argentina, Chile, Colombia, Costa Rica, Ecuador, México, Perú y Venezuela (Benavides y Alonso, 2012).

Basándonos en lo descrito por los autores y considerando lo valioso que resulta para mejorar la docencia el identificar los estilos de aprendizaje usados por estudiantes y docentes, es que se plantea el estudio de los estilos de aprendizaje de estudiantes y docentes de primer y segundo año de la carrera de Medicina Veterinaria de la Universidad Santo Tomás (Sede Concepción), mediante la aplicación del 'Cuestionario Honey y Alonso de Estilos de Aprendizaje' (CHAEA).

\section{Materiales y Métodos}

La población elegida para el estudio estuvo compuesta por la totalidad de estudiantes $(n=39)$ y docentes $(n=17)$ de primer y segundo año de la carrera de Medicina Veterinaria de la Universidad Santo Tomás, en la sede Concepción, ubicada en la VIII región, Chile. Las asignaturas que son dictadas en estos años están organizadas en un orden lógico y progresivo, de manera tal que los es- tudiantes adquieran los conocimientos básicos generales que sustenten una profesión con formación biológica relacionada con aspectos médicos.

El instrumento aplicado fue el 'Cuestionario Honey Alonso de Estilos de Aprendizaje' (CHAEA), validado por Honey y Alonso (Alonso et al., 1997). Antes de la aplicación del cuestionario, se le entregó a cada estudiante y docente, una carta de consentimiento informado, en la cual se dejaban estipulados los fines del presente estudio. El instrumento CHAEA consta de 80 preguntas (20 ítems para cada uno de los cuatros estilos) a las que se responde dicotómicamente manifestando si se está de acuerdo (signo +) o en desacuerdo (signo -). El cuestionario fue respondido en forma simultánea por todos los estudiantes sin tiempo límite para terminarlo. Asimismo, debían responder todas las preguntas. Los resultados de la aplicación del instrumento corresponden a una escala sumativa. Cada respuesta afirmativa dentro de cada estilo tiene el valor de 1 , de modo que la sumatoria máxima capaz de alcanzar cualquier Estilo de Aprendizaje es de 20.

Los datos obtenidos fueron analizados a través de un método estadístico de tipo descriptivo, expresándolo en cuadros y gráficos. Para las comparaciones de los grupos se utilizó la prueba estadística de Chi cuadrado, empleando un valor de $\mathrm{p}<0.05$ como nivel de significancia. 


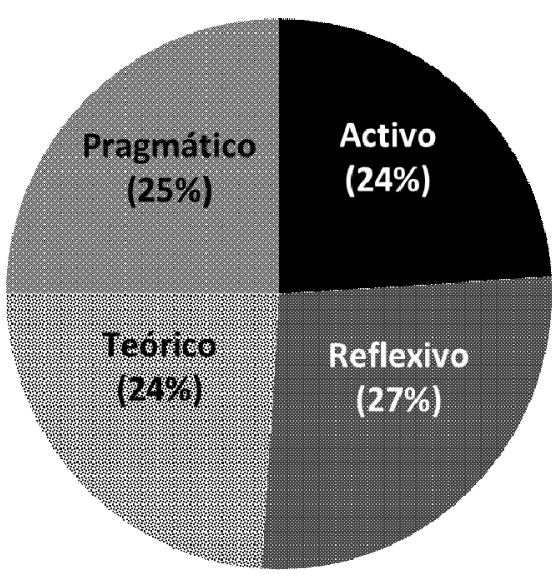

Estudiantes

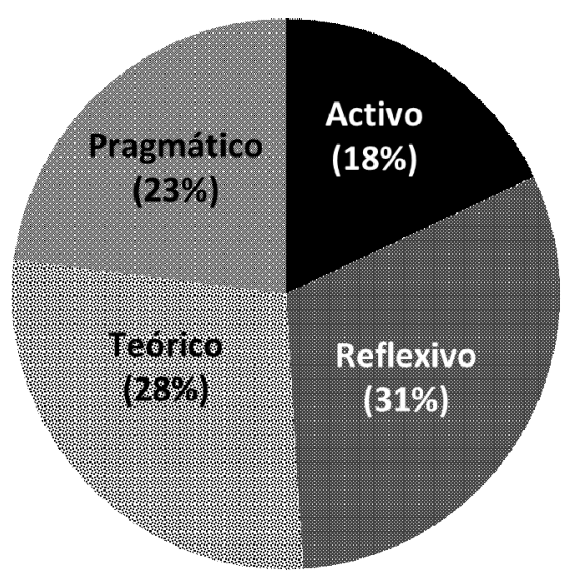

Docentes

Figura 1. Distribución porcentual de los estilos de aprendizaje del 'Cuestionario Honey Alonso de Estilos de Aprendizaje' (CHAEA) aplicado a los estudiantes $(n=39)$ y docentes $(n=17)$ de primer y segundo año de la carrera de Medicina Veterinaria en Concepción, Chile

\section{Resultados}

Los resultados indican que la preferencia en los estudiantes estuvo ligeramente dirigida al estilo de aprendizaje Reflexivo, con un puntaje promedio de presentación de 14.8 (máximo 20) (Cuadro 2). Sin embargo, los porcentajes de los estilos variaron entre $27 \%$ para el estilo Reflexivo y $24 \%$ para los estilos Activo y Teórico, no observándose diferencias estadísticas $(p=0.9994)$ entre ellos (Fig. 1).

En forma similar, en el caso de los docentes se observa una preferencia hacia el estilo de aprendizaje Reflexivo con un promedio de presentación de 16.1 (31\%), siendo el de menor promedio el estilo de aprendizaje Activo con 9.4 puntos en promedio (18\%), aunque sin diferencia estadística $(\mathrm{p}=0.9999)$ entre estilos (Fig. 1).

\section{Discusión}

Los resultados obtenidos son comparables a los descritos por Escanero-Marcén et al. (2013), quienes aplicaron el cuestionario CHAEA a estudiantes de la asignatura de fisiología, de la carrera de Medicina de la Universidad de Zaragoza, España. Resultados diferentes fueron obtenidos en estudiantes de la carrera de Medicina Veterinaria de la Universidad Austral, Valdivia, Chile (von Chrismar, 2005), donde se indica que la principal preferencia de estilo fue el Activo, a diferencia del Reflexivo que tuvo la menor frecuencia de presentación; así como en estudiantes de Medicina Veterinaria de la Universidad San Sebastián, Concepción, Chile, donde el estilo Teórico fue el que tuvo la mayor preferencia (Montero et al., 2011).

De acuerdo a los antecedentes bibliográficos analizados, era esperable que la población analizada hubiese presentado un estilo de aprendizaje preferentemente ActivoTeórico; no obstante, los resultados muestran una preferencia hacia lo Reflexivo. Von Chrismar (2005) plantea la posibilidad de que el sistema educativo se encuentre involucrado en la velocidad con que se mueve la sociedad. Esto, aunado a la protesta generalizada de los estudiantes secundarios en 2006 en Chile, protesta masiva y cohesionada que 
abogó por mejoras en la calidad de la educación, así como la constante transformación de las políticas de educación, donde se encuentran elementos de cambios y de continuidad (Valenzuela et al., 2008), permite que se observe esta evolución en los estilos de aprendizaje de Activo-Teórico a Reflexivo.

Estos cambios también se pueden observar en el estudio de Acuña et al. (2008), quienes compararon los estilos de aprendizaje de los estudiantes de las carreras del área de la salud, en la ciudad de Antofagasta, Chile, describiendo que el estilo Reflexivo fue el que alcanzó los mayores puntajes promedio. Los cambios generados por los movimientos estudiantiles el año 2006 producen un antes y un después en la manera que tienen los jóvenes chilenos de analizar la sociedad en su conjunto, por lo que el tipo de estudiante que fue sometido al instrumento CHAEA por von Chrismar en 2005 es diferente al estudiante encuestado en el presente estudio (cohortes 2012, 2013), lo cual sería la causa de la diferencia de los resultados obtenidos.

En relación a los docentes, los resultados concuerdan con los obtenidos por Coloma et al. (2008) en su estudio descriptivo de los estilos de aprendizaje de docentes universitarios de diversas carreras, donde los promedios alcanzados en orden de presentación fueron el estilo Reflexivo, Teórico, Pragmático y Activo. Por otro lado, en el estudio de Segura (2011), donde identifican y comparan las tendencias en los estilos de aprendizaje de estudiantes y profesores en contextos educativos venezolanos para utilizarlas como guía en la Mejora de la Gestión del Conocimiento, encontraron una tendencia en los docentes por el estilo de aprendizaje Reflexivo, seguido del Teórico.

Que la proporción de estilos de aprendizaje entre estudiantes y docentes sea relativamente similar es una ventaja en el proceso educativo, ya que esta similitud le facilita al profesor la meta de actuar como facilitador del aprendizaje, debido a la posibilidad de adecuar su estilo de enseñanza al modo de aprender del grupo. Asimismo, el estilo que posee el docente para aproximar la información al alumno es un modelo de aprendizaje que condiciona el estilo de aprendizaje del propio alumno (Adán, 2002).

\section{Conclusiones}

No se observa diferencia estadística entre estilos de aprendizaje de los estudiantes y docentes de primer y segundo año de la carrera de Medicina Veterinaria de la Universidad Santo Tomás, Concepción, Chile; sin embargo, se observa una preferencia moderada por el estilo de aprendizaje Reflexivo.

\section{Literatura Citada}

1. Acevedo C, Rocha F. 2011. Estilos de aprendizaje, género y rendimiento académico. Rev Estilos Aprendizaje 8: 7184.

2. Acuña O, Silva G, Maluenda R. 2008. Estilos de aprendizaje de los estudiantes de enfermería Universidad de Antofagasta. Rev Educ Cienc Salud 5(1): 26-32.

3. Adán MI. 2002. Estilos de aprendizaje, modalidades de bachillerato y rendimiento académico. Tesis Doctoral. España: Universidad Nacional de Educación a Distancia. $24 \mathrm{p}$.

4. Ahumada P. 2005. La evaluación auténtica: un sistema para la obtención de evidencias y vivencias de los aprendizajes. Perspectiva Educacional 45: 11-24.

5. Alonso C, Gallego D, Honey P. 1997. Los estilos de aprendizaje. Procedimiento de diagnóstico y mejora. $3^{\mathrm{a}}$ ed. Bilbao, España: Ed Mensajero. 221 p.

6. Benavides WIA, Alonso C. 2012. Los estilos de aprendizaje en los estudiantes de primer semestre de la Universidad Nacional Abierta y a Distancia UNADColombia. Rev Estilos Aprendizaje 10: 172-183. 
7. Coloma C, Manrique L, Revilla D, Tafur R. 2008. Estudio descriptivo de los estilos de aprendizaje de docentes universitarios. Rev Estilos Aprendizaje 1: 124-142.

8. Dunn R, Dunn K. 1984. La enseñanza y el estilo individual de aprendizaje. Madrid: Anaya. $422 \mathrm{p}$.

9. Escanero-Marcén JF, Soria MS, Escanero-Ereza ME, Guerra-Sánchez M. 2013. Influencia de los estilos de aprendizaje y la metacognición en el rendimiento académico de los estudiantes de fisiología. FEM 16(1): 23-29. doi: 10.4321/S2014-98322013000100005

10. Honey P, Mumford A. 1986. Using your learning styles. $2^{\text {nd }}$ ed. Maidenhead, Berkshire: Peter Honey. 88 p.

11. Juárez C, Rodríguez G, Luna E. 2012. El cuestionario de estilos de aprendizaje CHAEA y la escala de estrategias de aprendizaje ACRA como herramienta potencial para la tutoría académica. Rev Estilos Aprendizaje 10: 148-171.

12. Montero, E. Sepúlveda M, Contreras E. 2011. Estudio transversal de los estilos de aprendizaje y rendimiento académico en alumnos de $1^{\text {er. }}$ año de la carre- ra de Medicina Veterinaria. Rev Estilos Aprendizaje 7: 151-159.

13. Pantoja M, Duque, L, Correa J. 2013. Modelos de estilos de aprendizaje: una actualización para su revisión y análisis. Rev Colomb Educación 64: 79-105.

14. Segura M. 2011. Tendencias en los estilos de aprendizaje de estudiantes y profesores en instituciones educativas venezolanas de bachillerato y formación técnica superior. Rev Estilos Aprendizaje 7: 160-183.

15. Tarpy RM. 1999. Aprendizaje: teoría e investigación contemporáneas. Madrid: McGraw-Hill Interamericana. $712 \mathrm{p}$.

16. Valenzuela J, Labarrera P, Rodríguez P. 2008. Educación en Chile: entre la continuidad y la ruptura. Principales hitos de las políticas educativas. Rev Iberoamericana Educación 48: 129-145.

17. von Chrismar A. 2005. Identificación de los estilos de aprendizaje y propuesta de orientación pedagógica para estudiantes de la Universidad Austral de Chile. Tesis de Magíster. Valdivia, Chile: Universidad Austral de Chile. 96 p.

18. Witkin HA, Goodenoug DR. 1991. Estilos cognitivos. Naturaleza y orígenes. Madrid: Pirámide. 184 p. 